Article

\title{
Sufficient Conditions for Oscillation of Fourth-Order Neutral Differential Equations with Distributed Deviating Arguments
}

\author{
Omar Bazighifan ${ }^{1,2,+}\left(\mathbb{D}\right.$, Feliz Minhos ${ }^{3, *,+} \mathbb{D}$ and Osama Moaaz ${ }^{4,+}(\mathbb{D}$ \\ 1 Department of Mathematics, Faculty of Science, Hadhramout University, Hadhramout 50512, Yemen; \\ o.bazighifan@gmail.com \\ 2 Department of Mathematics, Faculty of Education, Seiyun University, Hadhramout 50512, Yemen \\ 3 Departamento de Matematica, Escola de Ciencias e Tecnologia, Centro de Investigacao em Matematica e \\ Aplicacoes (CIMA), Instituto de Investigacao e Formacao Avancada, Universidade de Evora, Rua Romao \\ Ramalho, 59, 7000-671 Evora, Portugal \\ 4 Department of Mathematics, Faculty of Science, Mansoura University, Mansoura 35516, Egypt; \\ o_moaaz@mans.edu.eg \\ * Correspondence: fminhos@uevora.pt \\ + These authors contributed equally to this work.
}

Received: 14 February 2020; Accepted: 8 April 2020; Published: 11 April 2020

check for updates

\begin{abstract}
Some new sufficient conditions are established for the oscillation of fourth order neutral differential equations with continuously distributed delay of the form $\left(r(t)\left(N_{x}^{\prime \prime \prime}(t)\right)^{\alpha}\right)^{\prime}+$ $\int_{a}^{b} q(t, \vartheta) x^{\beta}(\delta(t, \vartheta)) d \vartheta=0$, where $t \geq t_{0}$ and $N_{x}(t):=x(t)+p(t) x(\varphi(t))$. An example is provided to show the importance of these results.
\end{abstract}

Keywords: fourth-order differential equations; neutral delay; oscillation

\section{Introduction}

The theory of differential equations is an adequate mathematical apparatus for the simulation of processes and phenomena observed in biotechnology, neural networks, physics etc, see [1]. One area of active research in recent times is to study the sufficient criterion for oscillation of delay differential equations, see [1-28].

In this work, we establish the asymptotic behavior of fourth-order neutral differential equation of the form

$$
\left(r(t)\left(N_{x}^{\prime \prime \prime}(t)\right)^{\alpha}\right)^{\prime}+\int_{a}^{b} q(t, \vartheta) x^{\beta}(\delta(t, \vartheta)) d \vartheta=0,
$$

where $t \geq t_{0}$ and $N_{x}(t):=x(t)+p(t) x(\varphi(t))$. In this paper, we assume that:

A1: $\alpha$ and $\beta$ are a quotient of odd positive integers and $\beta \geq \alpha$;

A2: $r, p \in C\left[t_{0}, \infty\right), r(t)>0, r^{\prime}(t) \geq 0$ and $\int^{\infty} r^{-1 / \alpha}(s) \mathrm{d} s=\infty$;

A3: $q \in C\left(\left[t_{0}, \infty\right) \times(a, b), \mathbb{R}\right), q(t, \vartheta)>0,0 \leq p(t)<p_{0}<\infty$ and $q(t)$ is not identically zero for large $t$;

A4: $\varphi \in C^{1}\left[t_{0}, \infty\right), \delta \in p\left(\left[t_{0}, \infty\right) \times(a, b), \mathbb{R}\right), \varphi^{\prime}(t)>0, \varphi(t) \leq t, \lim _{t \rightarrow \infty} \varphi(t)=\lim _{t \rightarrow \infty} \delta(t, \vartheta)=$ $\infty$ and $\delta(t, \vartheta)$ has nondecreasing.

Definition 1. The function $x \in C^{3}\left[t_{y}, \infty\right), t_{y} \geq t_{0}$, is called a solution of (1), if $r(t)\left(N_{x}^{\prime \prime \prime}(t)\right)^{\alpha} \in$ $C^{1}\left[t_{y}, \infty\right)$, and $x(t)$ satisfies (1) on $\left[t_{y}, \infty\right)$. 
Definition 2. A solution of (1) is called oscillatory if it has arbitrarily large zeros on $\left[t_{x}, \infty\right)$, and otherwise is called to be nonoscillatory.

Definition 3. The Equation (1) is called oscillatory if every its solutions are oscillatory.

In the following, we discuss some important papers:

Chatzarakis et al. [9] proved the equation (1) where $\alpha=\beta$, is oscillatory, if

$$
\int_{t_{0}}^{\infty}\left(\omega(s)-\frac{2^{\alpha} r(s)}{\mu^{\alpha} s^{2 \alpha} \rho^{\alpha}(s)}\left(\frac{\rho^{\prime}(s)}{\alpha+1}\right)^{\alpha+1}\right) \mathrm{d} s=\infty,
$$

for some $\mu \in(0,1)$ and

$$
\int_{t_{0}}^{\infty}\left(\vartheta(s)\left(\int_{t}^{\infty}(Q(v))^{\frac{1}{\alpha}} r^{\frac{-1}{\alpha}}(v) d v\right)-\frac{\theta_{+}^{\prime 2}(s)}{4 \theta(s)}\right) \mathrm{d} s=\infty,
$$

where $\mathscr{\omega}(t):=k \rho(t) Q(t)(1-p(\delta(t, a)))^{\alpha}(\delta(t, a) \backslash t)^{3 \alpha}$ and $\rho, \theta \in C^{1}\left(\left[v_{0}, \infty\right),(0, \infty)\right)$.

Moaaz et al. in [19] extended the Riccati transformation to obtain new oscillatory criteria for (1) as condition

$$
\int_{t_{0}}^{\infty}\left[\theta(s) Q(s)-\frac{1}{\lambda 4}\left(\frac{\theta^{\prime}(s)}{\theta(s)}\right)^{2}\right] \mathrm{d} s=\infty,
$$

where $\lambda \in(0,1)$ and a function $\theta \in C^{1}\left(\left[v_{0}, \infty\right),(0, \infty)\right)$.

Authors in [24] studied oscillatory behavior of equation

$$
N_{x}^{(n)}(t)+q(t) x(\delta(t))=0
$$

where $n$ is even, they proved it oscillatory by using the Riccati transformation if either

$$
\lim \inf _{t \rightarrow \infty} \int_{\varphi(t)}^{t} Q(s) \mathrm{d} s>\frac{(n-1) !}{\mathrm{e}}
$$

or

$$
\lim \sup _{t \rightarrow \infty} \int_{\varphi(t)}^{t} Q(s) \mathrm{d} s>(n-1) !,
$$

where $Q(t):=\varphi^{n-1}(t)(1-p(\varphi(t))) q(t)$.

Xing et al. [22] proved that the even-order differential equation

$$
\left(r(t)\left(N_{x}^{(n-1)}(t)\right)^{\alpha}\right)^{\prime}+q(t) x^{\beta}(\delta(t))=0,
$$

is oscillatory, if

$$
\left(\delta^{-1}(t)\right)^{\prime} \geq \delta_{0}>0, \varphi^{\prime}(t) \geq \varphi_{0}>0, \varphi^{-1}(\delta(t))<t
$$

and

$$
\lim \inf _{t \rightarrow \infty} \int_{\varphi^{-1}(\delta(t))}^{t} \frac{\widehat{q}(s)}{r(s)}\left(s^{n-1}\right)^{\alpha} \mathrm{d} s>\frac{\left(\frac{1}{\delta_{0}}+\frac{p_{0}^{\alpha}}{\delta_{0} \varphi_{0}}\right)}{\mathrm{e}((n-1) !)^{-\alpha}},
$$

where $\widehat{q}(t):=\min \left\{q\left(\delta^{-1}(t)\right), q\left(\delta^{-1}(\varphi(t))\right)\right\}$ and $n$ is even.

To prove this, we apply the previous results to the equation

$$
(x(t)+p x(\varphi t))^{(n)}+b x(\delta t)=0, t \geq 1,
$$

where $n=4, p=7 / 8, \varphi=1 / \mathrm{e}, \delta=1 / \mathrm{e}^{2}$ and $b=q_{0} / v^{4}$, we find: 
1. By applying condition (3) in (5), we find

$$
q_{0}>3561.9
$$

2. By applying condition (4) in (5), we get

$$
q_{0}>3008.5
$$

Hence, [22] improved the results in [24].

Thus, the motivation in studying this paper is complement results in [9] and improve results [22,24].

By using the Riccati transformations, we establish a new oscillation criterion for a class of fourth-order neutral differential equations (1). An example is provided to illustrate the main results.

\section{Some Auxiliary Lemmas}

We shall employ the following lemmas

Lemma 1 ([3]). Let $x \in C^{n}\left(\left[t_{0}, \infty\right),(0, \infty)\right)$. Assume that $x^{(n)}(t)$ is of fixed sign and not identically zero on $\left[t_{0}, \infty\right)$ and there exists $a t_{1} \geq t_{0}$ such that $x^{(n-1)}(t) x^{(n)}(t) \leq 0$ for all $t \geq t_{1}$. If $\lim _{t \rightarrow \infty} x(t) \neq 0$, then for every $\mu \in(0,1)$ there exists $t_{\mu} \geq t_{1}$ such that

$$
x(t) \geq \frac{\mu}{(n-1) !} t^{n-1}\left|x^{(n-1)}(t)\right| \text { for } t \geq t_{\mu} .
$$

Lemma 2 ([16]). Let the function $x$ satisfies $x^{(i)}(t)>0, i=0,1, \ldots, n$, and $x^{(n+1)}(t)<0$, then

$$
\frac{x(t)}{t^{n} / n !} \geq \frac{x^{\prime}(t)}{t^{n-1} /(n-1) !} .
$$

Lemma 3 ([4]). Assume that $x, v \geq 0$ and $\alpha \geq 1$ is a positive real number. Then

$$
(x+v)^{\alpha} \leq 2^{\alpha-1}\left(x^{\alpha}+v^{\alpha}\right)
$$

and

$$
(x+v)^{\beta} \leq x^{\beta}+v^{\beta}, \text { for } \beta \leq 1 .
$$

Lemma 4 ([9]). Assume that $x$ is an eventually positive solution of (1). Then, there exist two possible cases:

$$
\begin{aligned}
& \left(\mathbf{S}_{1}\right) N_{x}^{(\kappa)}(t)>0 \text { for } \kappa=0,1,2,3 ; \\
& \left(\mathbf{S}_{2}\right) N_{x}(t)>0, N_{x}^{\prime}(t)>0, N_{x}^{\prime \prime}(t)<0 \text { and } N_{x}^{\prime \prime \prime}(t)>0,
\end{aligned}
$$

for $t \geq t_{1}$, where $t_{1} \geq t_{0}$ is sufficiently large. 
Notation 1. We consider the following notations:

$$
\begin{aligned}
& p_{1}(t)=\frac{1}{p\left(\varphi^{-1}(t)\right)}\left(1-\frac{\left(\varphi^{-1}\left(\varphi^{-1}(t)\right)\right)^{3}}{\left(\varphi^{-1}(t)\right)^{3} p\left(\varphi^{-1}\left(\varphi^{-1}(t)\right)\right)}\right) \\
& p_{2}(t)=\frac{1}{p\left(\varphi^{-1}(t)\right)}\left(1-\frac{\left(\varphi^{-1}\left(\varphi^{-1}(t)\right)\right)}{\left(\varphi^{-1}(t)\right) p\left(\varphi^{-1}\left(\varphi^{-1}(t)\right)\right)}\right) \\
& \Psi(t)=M_{1}^{\beta-\alpha} \theta(t) \int_{a}^{b} q(t, \vartheta) p_{1}^{\beta}(\delta(t, \vartheta)) d \vartheta \\
& \tilde{R}(t)=\int_{a}^{b}\left(\frac{\mu\left(\varphi^{-1}(\eta(t, \vartheta))\right)^{3}}{6}\right)^{\beta} q(t, \vartheta) p_{1}^{\beta}(\eta(t, \vartheta)) r^{-\beta / \alpha}\left(\varphi^{-1}(\eta(t, \vartheta))\right) \mathrm{d} \vartheta \\
& R(t)=\int_{t}^{\infty}\left(\frac{1}{r(\varrho)} \int_{\varrho}^{\infty}\left(\int_{a}^{b} q(s, \vartheta)\left(\frac{\varphi^{-1}(\sigma(s, \vartheta))}{s}\right)^{\beta} \mathrm{d} \vartheta\right) \mathrm{d} s\right)^{1 / \alpha} \mathrm{d} \varrho,
\end{aligned}
$$

and

$$
\Phi(t):=p_{2}^{\beta / \alpha} \theta_{1}(t) M_{2}^{(\beta-\alpha) / \alpha} \int_{t}^{\infty}\left(\frac{1}{r(\varrho)} \int_{\varrho}^{\infty}\left(\int_{a}^{b} q(s, \vartheta)\left(\frac{\varphi^{-1}(\delta(s, \vartheta))}{s}\right)^{\beta} \mathrm{d} \vartheta\right) \mathrm{d} s\right)^{1 / \alpha} \mathrm{d} \varrho .
$$

\section{Main Results}

In this part, we will discuss some oscillation criteria for Equation (1).

Lemma 5. Assume that $x$ is an eventually positive solution of (1) and

$$
\left(\varphi^{-1}\left(\varphi^{-1}(t)\right)\right)^{3}<\left(\varphi^{-1}(t)\right)^{3} p\left(\varphi^{-1}\left(\varphi^{-1}(t)\right)\right) .
$$

Then

$$
x(t) \geq \frac{1}{p\left(\varphi^{-1}(t)\right)}\left(N_{x}\left(\varphi^{-1}(t)\right)-\frac{1}{p\left(\varphi^{-1}\left(\varphi^{-1}(t)\right)\right)} N_{x}\left(\varphi^{-1}\left(\varphi^{-1}(t)\right)\right)\right) .
$$

Proof. Let $x$ be an eventually positive solution of (1) on $\left[t_{0}, \infty\right)$. From the definition of $z(t)$, we see that

$$
p(t) x(\varphi(t))=N_{x}(t)-x(t),
$$

and so

$$
p\left(\varphi^{-1}(t)\right) x(t)=N_{x}\left(\varphi^{-1}(t)\right)-x\left(\varphi^{-1}(t)\right) .
$$

Repeating the same process, we obtain

$$
x(t)=\frac{1}{p\left(\varphi^{-1}(t)\right)}\left(N_{x}\left(\varphi^{-1}(t)\right)-\left(\frac{N_{x}\left(\varphi^{-1}\left(\varphi^{-1}(t)\right)\right)}{p\left(\varphi^{-1}\left(\varphi^{-1}(t)\right)\right)}-\frac{x\left(\varphi^{-1}\left(\varphi^{-1}(t)\right)\right)}{p\left(\varphi^{-1}\left(\varphi^{-1}(t)\right)\right)}\right)\right),
$$

which yields

$$
x(t) \geq \frac{N_{x}\left(\varphi^{-1}(t)\right)}{p\left(\varphi^{-1}(t)\right)}-\frac{1}{p\left(\varphi^{-1}(t)\right)} \frac{N_{x}\left(\varphi^{-1}\left(\varphi^{-1}(t)\right)\right)}{p\left(\varphi^{-1}\left(\varphi^{-1}(t)\right)\right)} .
$$

Thus, (7) holds. This completes the proof. 
Theorem 1. Let $\delta(t) \leq \varphi(t)$ and (6) holds. If there exist positive functions $\theta, \theta_{1} \in C^{1}\left(\left[t_{0}, \infty\right), \mathbb{R}\right)$ such that

$$
\int_{t_{0}}^{\infty}\left(\Psi(s)-\frac{2^{\alpha}}{(\alpha+1)^{\alpha+1}} \frac{r\left(\varphi^{-1}(\delta(s, a))\right)\left(\theta^{\prime}(s)\right)^{\alpha+1}}{\left(\mu_{1} \theta(s)\left(\varphi^{-1}(\delta(s, a))\right)^{\prime}(\delta(s, a))^{\prime}\left(\varphi^{-1}(\delta(s, a))\right)^{2}\right)^{\alpha}}\right) \mathrm{d} s=\infty
$$

and

$$
\int_{t_{0}}^{\infty}\left(\Phi(s)-\frac{\left(\theta_{1}^{\prime}(s)\right)^{2}}{4 \theta_{1}(s)}\right) \mathrm{d} s=\infty
$$

for some $\mu_{1} \in(0,1)$ and every $M_{1}, M_{2}>0$, then (1) is oscillatory.

Proof. Let $x$ be a non-oscillatory solution of (1) on $\left[t_{0}, \infty\right)$. Without loss of generality, we can assume that $x$ is eventually positive. It follows from Lemma 4 that there exist two possible cases $\left(\mathbf{S}_{1}\right)$ and $\left(\mathbf{S}_{2}\right)$.

Let $\left(\mathbf{S}_{1}\right)$ holds. From Lemma 2, we obtain $N_{x}(t) \geq \frac{1}{3} t N_{x}^{\prime}(t)$ and hence the function $t^{-3} N_{x}(t)$ is nonincreasing, which with the fact that $\varphi^{-1}(t) \leq \varphi^{-1}\left(\varphi^{-1}(t)\right)$ gives

$$
\left(\varphi^{-1}(t)\right)^{3} N_{x}\left(\varphi^{-1}\left(\varphi^{-1}(t)\right)\right) \leq\left(\varphi^{-1}\left(\varphi^{-1}(t)\right)\right)^{3} N_{x}\left(\varphi^{-1}(t)\right) .
$$

From (7) and (10), we get that

$$
\begin{aligned}
x(t) & \geq \frac{N_{x}\left(\varphi^{-1}(t)\right)}{p\left(\varphi^{-1}(t)\right)}\left(1-\frac{\left(\varphi^{-1}\left(\varphi^{-1}(t)\right)\right)^{n-1}}{\left(\varphi^{-1}(t)\right)^{n-1} p\left(\varphi^{-1}\left(\varphi^{-1}(t)\right)\right)}\right) \\
& \geq p_{1}(t) N_{x}\left(\varphi^{-1}(t)\right) .
\end{aligned}
$$

From (1) and (11), we obtain

$$
\left(r(t)\left(N_{x}^{\prime \prime \prime}(t)\right)^{\alpha}\right)^{\prime}+\int_{a}^{b} q(t, \vartheta) p_{1}^{\beta}(\delta(t, \vartheta)) N_{x}^{\beta}\left(\varphi^{-1}(\delta(t, \vartheta))\right) d \vartheta \leq 0 .
$$

Since $\delta(t, \xi)$ is nondecreasing with respect tos, we get $\delta(t, \vartheta) \geq \delta(t, a)$ for $\xi \in(a, b)$ and so

$$
\left(r(t)\left(N_{x}^{\prime \prime \prime}(t)\right)^{\alpha}\right)^{\prime}+N_{x}^{\beta}\left(\varphi^{-1}(\delta(t, a))\right) \int_{a}^{b} q(t, \vartheta) p_{1}^{\beta}(\delta(t, \vartheta)) d \vartheta \leq 0 .
$$

Next, we define a function $\omega$ by

$$
\omega(t):=\theta(t) \frac{r(t)\left(N_{x}^{\prime \prime \prime}(t)\right)^{\alpha}}{N_{x}^{\alpha}\left(\varphi^{-1}(\delta(t, a))\right)}>0 .
$$

Differentiating and using (12), we obtain

$$
\begin{aligned}
\omega^{\prime}(t) \leq & \frac{\theta^{\prime}(t)}{\theta(t)} \omega(t)-\theta(t) N_{x}^{\beta-\alpha}\left(\varphi^{-1}(\delta(t, a))\right) \int_{a}^{b} q(t, \vartheta) p_{1}^{\beta}(\delta(t, \vartheta)) d \vartheta \\
& -\alpha \theta(t) \frac{r(t)\left(N_{x}^{\prime \prime \prime}(t)\right)^{\alpha}\left(\varphi^{-1}(\delta(t, a))\right)^{\prime}(\delta(t, a))^{\prime} N_{x}^{\prime}\left(\varphi^{-1}(\delta(t, a))\right)}{N_{x}^{\alpha+1}\left(\varphi^{-1}(\delta(t, a))\right)} .
\end{aligned}
$$

Recalling that $r(t)\left(N_{x}^{\prime \prime \prime}(t)\right)^{\alpha}$ is decreasing, we get

$$
r\left(\varphi^{-1}(\delta(t, a))\right)\left(N_{x}^{\prime \prime \prime}\left(\varphi^{-1}(\delta(t, a))\right)\right)^{\alpha} \geq r(t)\left(N_{x}^{\prime \prime \prime}(t)\right)^{\alpha} .
$$


This yields

$$
\left(N_{x}^{\prime \prime \prime}\left(\varphi^{-1}(\delta(t, a))\right)\right)^{\alpha} \geq \frac{r(t)}{r\left(\varphi^{-1}(\delta(t, a))\right)}\left(N_{x}^{\prime \prime \prime}(t)\right)^{\alpha} .
$$

It follows from Lemma 1 that

$$
N_{x}^{\prime}\left(\varphi^{-1}(\delta(t, a))\right) \geq \frac{\mu_{1}}{2}\left(\varphi^{-1}(\delta(t, a))\right)^{2} N_{x}^{\prime \prime \prime}\left(\varphi^{-1}(\delta(t, a))\right),
$$

for all $\mu_{1} \in(0,1)$. Thus, by (13)-(15), we get

$$
\begin{aligned}
\omega^{\prime}(t) \leq & \frac{\theta^{\prime}(t)}{\theta(t)} \omega(t)-\theta(t) N_{x}^{\beta-\alpha}\left(\varphi^{-1}(\delta(t, a))\right) \int_{a}^{b} q(t, \vartheta) p_{1}^{\beta}(\delta(t, \vartheta)) d \vartheta \\
& -\alpha \theta(t) \frac{\mu_{1}}{2}\left(\frac{r(t)}{r\left(\varphi^{-1}(\delta(t, a))\right)}\right)^{1 / \alpha} \frac{r(t)\left(N_{x}^{\prime \prime \prime}(t)\right)^{\alpha+1}\left(\varphi^{-1}(\delta(t, a))\right)^{\prime}(\delta(t, a))^{\prime}\left(\varphi^{-1}(\delta(t, a))\right)^{2}}{N_{x}^{\alpha+1}\left(\varphi^{-1}(\delta(t, a))\right)}
\end{aligned}
$$

Hence,

$$
\begin{aligned}
\omega^{\prime}(t) \leq & \frac{\theta^{\prime}(t)}{\theta(t)} \omega(t)-\theta(t) N_{x}^{\beta-\alpha}\left(\varphi^{-1}(\delta(t, a))\right) \int_{a}^{b} q(t, \vartheta) p_{1}^{\beta}(\delta(t, \vartheta)) d \vartheta \\
& -\alpha \frac{\mu_{1}}{2}\left(\frac{r(t)}{r\left(\varphi^{-1}(\delta(t, a))\right)}\right)^{1 / \alpha} \frac{\left(\varphi^{-1}(\delta(t, a))\right)^{\prime}(\delta(t, a))^{\prime}\left(\varphi^{-1}(\delta(t, a))\right)^{2}}{(r \theta)^{1 / \alpha}(t)} \omega^{\frac{\alpha+1}{\alpha}}(t) .
\end{aligned}
$$

Since $N_{x}^{\prime}(t)>0$, there exist a $t_{2} \geq t_{1}$ and a constant $M>0$ such that

$$
N_{x}(t)>M,
$$

for all $t \geq t_{2}$. Using the inequality

$$
U x-V x^{(\beta+1) / \beta} \leq \frac{\beta^{\beta}}{(\beta+1)^{\beta+1}} \frac{U^{\beta+1}}{V^{\beta}}, V>0,
$$

with

$$
U=\frac{\theta^{\prime}(t)}{\theta(t)}, V=\alpha \frac{\mu_{1}}{2}\left(\frac{r(t)}{r\left(\varphi^{-1}(\delta(t, a))\right)}\right)^{1 / \alpha} \frac{\left(\varphi^{-1}(\delta(t, a))\right)^{\prime}(\delta(t, a))^{\prime}\left(\varphi^{-1}(\delta(t, a))\right)^{2}}{(r \theta)^{1 / \alpha}(t)}
$$

and $x=\omega$, we get

$$
\omega^{\prime}(t) \leq-\Psi(t)+\frac{2^{\alpha}}{(\alpha+1)^{\alpha+1}} \frac{r\left(\varphi^{-1}(\delta(t, a))\right)\left(\theta^{\prime}(t)\right)^{\alpha+1}}{\left(\mu_{1} \theta(t)\left(\varphi^{-1}(\delta(t, a))\right)^{\prime}(\delta(t, a))^{\prime}\left(\varphi^{-1}(\delta(t, a))\right)^{2}\right)^{\alpha}} .
$$

This implies that

$$
\int_{t_{1}}^{t}\left(\Psi(s)-\frac{2^{\alpha}}{(\alpha+1)^{\alpha+1}} \frac{r\left(\varphi^{-1}(\delta(t, a))\right)\left(\theta^{\prime}(t)\right)^{\alpha+1}}{\left(\mu_{1} \theta(t)\left(\varphi^{-1}(\delta(t, a))\right)^{\prime}(\delta(t, a))^{\prime}\left(\varphi^{-1}(\delta(t, a))\right)^{2}\right)^{\alpha}}\right) \mathrm{d} s \leq \omega\left(t_{1}\right),
$$

which contradicts (8).

In the case where $\left(\mathbf{S}_{2}\right)$ satisfies, by using Lemma 2, we find that

$$
N_{x}(t) \geq t N_{x}^{\prime}(t)
$$


Axioms 2020, 9, 39

7 of 11

and hence $\left(t^{-1} N_{x}(t)\right)^{\prime} \leq 0$. Therefore,

$$
\varphi^{-1}(t) N_{x}\left(\varphi^{-1}\left(\varphi^{-1}(t)\right)\right) \leq \varphi^{-1}\left(\varphi^{-1}(t)\right) N_{x}\left(\varphi^{-1}(t)\right) .
$$

From (7) and (18), we have

$$
\begin{aligned}
x(t) & \geq \frac{1}{p\left(\varphi^{-1}(t)\right)}\left(1-\frac{\left(\varphi^{-1}\left(\varphi^{-1}(t)\right)\right)}{\left(\varphi^{-1}(t)\right) p\left(\varphi^{-1}\left(\varphi^{-1}(t)\right)\right)}\right) N_{x}\left(\varphi^{-1}(t)\right) \\
& =p_{2}(t) N_{x}\left(\varphi^{-1}(t)\right),
\end{aligned}
$$

which with (1) gives

$$
\left(r(t)\left(N_{x}^{\prime \prime \prime}(t)\right)^{\alpha}\right)^{\prime} \leq-\int_{a}^{b} q(t, \vartheta) p_{2}^{\beta}(\delta(t, \vartheta)) N_{x}^{\beta}\left(\varphi^{-1}(\delta(t, \vartheta))\right) d \vartheta .
$$

Integrating this inequality from $t$ to $\varrho$, we obtain

$r(\varrho)\left(N_{x}^{\prime \prime \prime}(\varrho)\right)^{\alpha}-r(t)\left(N_{x}^{\prime \prime \prime}(t)\right)^{\alpha} \leq-\int_{t}^{\varrho}\left(\int_{a}^{b} q(t, \vartheta) p_{2}^{\beta}(\delta(t, \vartheta)) N_{x}^{\beta}\left(\varphi^{-1}(\delta(t, \vartheta))\right) \mathrm{d} \vartheta\right) \mathrm{d} s$.

From (17), we get that

$$
N_{x}\left(\varphi^{-1}(\delta(t, \vartheta))\right) \geq \frac{\varphi^{-1}(\delta(t, \vartheta))}{t} N_{x}(t) .
$$

Letting $\varrho \rightarrow \infty$ in (19) and using (20), we obtain

$$
r(t)\left(N_{x}^{\prime \prime \prime}(t)\right)^{\alpha} \geq p_{2}^{\beta}(\delta(t, a)) N_{x}^{\beta}(t) \int_{t}^{\infty}\left(\int_{a}^{b} q(s, \vartheta)\left(\frac{\varphi^{-1}(\delta(s, \vartheta))}{s}\right)^{\beta} \mathrm{d} \vartheta\right) \mathrm{d} s .
$$

Integrating this inequality again from $t$ to $\infty$, we get

$$
N_{x}^{\prime \prime}(t) \leq-p_{2}^{\beta / \alpha} N_{x}^{\beta / \alpha}(t) \int_{t}^{\infty}\left(\frac{1}{r(\varrho)} \int_{\varrho}^{\infty}\left(\int_{a}^{b} q(s, \vartheta)\left(\frac{\varphi^{-1}(\delta(s, \vartheta))}{s}\right)^{\beta} \mathrm{d} \vartheta\right) \mathrm{d} s\right)^{1 / \alpha} \mathrm{d} \varrho,
$$

for all $\mu_{2} \in(0,1)$.

Now, we define

$$
w(t)=\theta_{1}(t) \frac{N_{x}^{\prime}(t)}{N_{x}(t)} .
$$

Then $w(t)>0$ for $t \geq t_{1}$. By differentiating $w$ and using (21), we find

$$
\begin{aligned}
w^{\prime}(t)= & \frac{\theta_{1}^{\prime}(t)}{\theta_{1}(t)} w(t)+\theta_{1}(t) \frac{N_{x}^{\prime \prime}(t)}{N_{x}(t)}-\theta_{1}(t)\left(\frac{N_{x}^{\prime}(t)}{N_{x}(t)}\right)^{2} \\
\leq & \frac{\theta_{1}^{\prime}(t)}{\theta_{1}(t)} w(t)-\frac{1}{\theta_{1}(t)} w^{2}(t) \\
& -p_{2}^{\beta / \alpha} \theta_{1}(t) N_{x}^{\beta / \alpha-1}(t) \int_{t}^{\infty}\left(\frac{1}{r(\varrho)} \int_{\varrho}^{\infty}\left(\int_{a}^{b} q(s, \vartheta)\left(\frac{\varphi^{-1}(\delta(s, \vartheta))}{s}\right)^{\beta} \mathrm{d} \vartheta\right) \mathrm{d} s\right)^{1 / \alpha} \mathrm{d} \varrho .
\end{aligned}
$$

Thus, we obtain

$$
w^{\prime}(t) \leq-\Phi(t)+\frac{\theta_{1}^{\prime}(t)}{\theta_{1}(t)} w(t)-\frac{1}{\theta_{1}(t)} w^{2}(t)
$$


and so

$$
w^{\prime}(t) \leq-\Phi(t)+\frac{\left(\theta_{1}^{\prime}(t)\right)^{2}}{4 \theta_{1}(t)}
$$

Then, we get

$$
\int_{t_{1}}^{t}\left(\Phi(s)-\frac{\left(\theta^{\prime}(t)\right)^{2}}{4 \theta(t)}\right) \mathrm{d} s \leq w\left(t_{1}\right)
$$

which contradicts (9). This completes the proof.

Theorem 2. Let

$$
\frac{\left(\varphi^{-1}\left(\varphi^{-1}(t)\right)\right)^{n-1}}{\left(\varphi^{-1}(t)\right)^{n-1} p\left(\varphi^{-1}\left(\varphi^{-1}(t)\right)\right)} \leq 1 .
$$

Suppose that there exist positive functions $\eta, \sigma \in p^{1}\left(\left[t_{0}, \infty\right), \mathbb{R}\right)$ satisfying

$\eta(t) \leq \delta(t), \eta(t)<\varphi(t), \sigma(t) \leq \delta(t), \sigma(t)<\varphi(t), \sigma^{\prime}(t) \geq 0$ and $\lim _{t \rightarrow \infty} \eta(t)=\lim _{t \rightarrow \infty} \sigma(t)=\infty$.

If the equations

$$
\psi^{\prime}(t)+\tilde{R}(t) \psi^{\beta / \alpha}\left(\varphi^{-1}(\eta(t, a))\right)=0
$$

and

$$
\phi^{\prime}(t)+p_{2}^{\beta / \alpha}\left(\varphi^{-1}(\sigma(t, a))\right)^{\beta / \alpha} R(t) \phi^{\beta / \alpha}\left(\varphi^{-1}(\sigma(t, a))\right)=0
$$

are oscillatory, then (1) is oscillatory.

Proof. Let $x$ be a non-oscillatory solution of (1) on $\left[t_{0}, \infty\right)$. Without loss of generality, we suppose that $x>0$. From Lemma 4 , we find there exist two possible cases $\left(\mathbf{S}_{1}\right)$ and $\left(\mathbf{S}_{2}\right)$.

Assume that Case $\left(\mathbf{S}_{1}\right)$ holds. From Theorem 1, we get that (12) holds. Since $\eta(t) \leq \delta(t)$ and $z^{\prime}(t)>0$, we obtain

$$
\left(r(t)\left(N_{x}^{\prime \prime \prime}(t)\right)^{\alpha}\right)^{\prime} \leq-\int_{a}^{b} q(t, \vartheta) p_{1}^{\beta}(\eta(t, \vartheta)) N_{x}^{\beta}\left(\varphi^{-1}(\eta(t, \vartheta))\right) \mathrm{d} \vartheta .
$$

Now, by using Lemma 1, we have

$$
N_{x}(t) \geq \frac{\mu}{6} t^{3} N_{x}^{\prime \prime \prime}(t)
$$

for some $\mu \in(0,1)$. It follows from (26) and (27) that, for all $\mu \in(0,1)$,

$$
\left(r(t)\left(N_{x}^{\prime \prime \prime}(t)\right)^{\alpha}\right)^{\prime}+\int_{a}^{b}\left(\frac{\mu\left(\varphi^{-1}(\eta(t, \vartheta))\right)^{3}}{6}\right)^{\beta} q(t, \vartheta) p_{1}^{\beta}(\eta(t, \vartheta))\left(N_{x}^{\prime \prime \prime}\left(\varphi^{-1}(\eta(t, \vartheta))\right)\right)^{\beta} \mathrm{d} \vartheta \leq 0 .
$$

Thus, we choose

$$
\psi(t)=r(t)\left(N_{x}^{\prime \prime \prime}(t)\right)^{\alpha} .
$$

So, we find that $\psi$ is a positive solution of the inequality

$$
\psi^{\prime}(t)+\tilde{R}(t) \psi^{\beta / \alpha}\left(\varphi^{-1}(\eta(t, a))\right) \leq 0 .
$$

Using (see ([15] Theorem 1)), we see (24) also has a positive solution, a contradiction.

Suppose that Case $\left(\mathbf{S}_{2}\right)$ holds. From Theorem 1 , we get that (21) holds. Since $\sigma(t) \leq \delta(t)$ and $N_{x}^{\prime}(t)>0$, we have that 


$$
N_{x}^{\prime \prime}(t) \leq-p_{2}^{\beta / \alpha} N_{x}^{\beta / \alpha}\left(\varphi^{-1}(\sigma(t, a))\right) \int_{t}^{\infty}\left(\frac{1}{r(\varrho)} \int_{\varrho}^{\infty}\left(\int_{a}^{b} q(s, \vartheta)\left(\frac{\varphi^{-1}(\sigma(s, \vartheta))}{s}\right)^{\beta} \mathrm{d} \vartheta\right) \mathrm{d} s\right)^{1 / \alpha} \mathrm{d} \varrho
$$

Using Lemma 2, we get that

$$
N_{x}(t) \geq t N_{x}^{\prime}(t)
$$

From (18) and (29), we obtain

$$
N_{x}^{\prime \prime}(t) \leq-p_{2}^{\beta / \alpha}\left(N_{x}^{\prime}\left(\varphi^{-1}(\sigma(t, a))\right)\right)^{\beta / \alpha}\left(\varphi^{-1}(\sigma(t, a))\right)^{\beta / \alpha} R(t) .
$$

Now, we choose $\phi(t):=N_{x}^{\prime}(t)$, thus, we find that $\phi$ is a positive solution of

$$
\phi^{\prime}(t)+p_{2}^{\beta / \alpha}\left(\varphi^{-1}(\sigma(t, a))\right)^{\beta / \alpha} R(t) \phi^{\beta / \alpha}\left(\varphi^{-1}(\sigma(t, a))\right) \leq 0 .
$$

Using (see ([15] Theorem 1)), we see (25) also has a positive solution, a contradiction. The proof is complete.

Example 1. Consider the differential equation

$$
\left(\left[x(t)+\frac{1}{2} x\left(\frac{t}{3}\right)\right]^{\prime \prime \prime}\right)^{\prime}+\int_{0}^{1}\left(\frac{q_{0}}{t^{4}}\right) \vartheta x\left(\frac{t-\xi}{2}\right) d \vartheta=0,
$$

where $q_{0}>0$ is a constant. Let $\alpha=\beta=1, r(t)=1, p(t)=1 / 2, \varphi(t)=t / 3, \varphi^{-1}(t)=3 t, \delta(t, a)=$ $t / 2, q(t, \vartheta)=\left(q_{0} \backslash t^{4}\right) \vartheta$.

Thus, by using Theorem 1, then Equation (31) is oscillatory.

Remark 1. By applying our results in (5), we see that our results improve [22,24].

Remark 2. One can easily see that the results obtained in [24] cannot be applied to conditions in Theorem 1, so our results are new.

\section{Conclusions}

In this work, our method is based on using the Riccati transformations to get some oscillation criteria of (1). There are numerous results concerning the oscillation criteria of fourth order equations, which include various forms of criteria as Hille/Nehari, Philos, etc. This allows us to obtain also various criteria for the oscillation of (1). Further, we can try to get some oscillation criteria of (1) if $N_{x}(t):=x(t)-p(t) x(\varphi(t))$ in the future work.

Author Contributions: The authors claim to have contributed equally and significantly in this paper. All authors have read and agreed to the published version of the manuscript.

Funding: The authors received no direct funding for this work.

Acknowledgments: The authors thank the reviewers for for their useful comments, which led to the improvement of the content of the paper.

Conflicts of Interest: There are no competing interests between the authors. 


\section{References}

1. Hale, J.K. Theory of Functional Differential Equations; Springer: New York, NY, USA, 1977.

2. Agarwal, R.P.; Bohner, M.; Li, T.; Zhang, C. A new approach in the study of oscillatory behavior of even-order neutral delay diferential equations. Appl. Math. Comput. 2013, 225, 787-794.

3. Agarwal, R.; Grace, S.; O'Regan, D. Oscillation Theory for Difference and Functional Differential Equations; Kluwer Acad. Publ.: Dordrecht, The Netherlands, 2000.

4. Baculikova, B.; Dzurina, J. Oscillation theorems for second-order nonlinear neutral differential equations. Comput. Math. Appl. 2011, 62, 4472-4478. [CrossRef]

5. Bazighifan, O.; Cesarano, C. Some New Oscillation Criteria for Second-Order Neutral Differential Equations with Delayed Arguments. Mathematics 2019, 7, 619. [CrossRef]

6. Bazighifan, O.; Elabbasy, M.E.; Moaaz, O. Oscillation of higher-order differential equations with distributed delay. J. Inequal. Appl. 2019, 55, 1-9. [CrossRef]

7. Bazighifan, O.; Postolache, M. An improved conditions for oscillation of functional nonlinear differential equations. Mathematics 2020, 8, 552. [CrossRef]

8. Bazighifan, O. An Approach for Studying Asymptotic Properties of Solutions of Neutral Differential Equations. Symmetry 2020, 12, 555. [CrossRef]

9. Chatzarakis, G.E.; Elabbasy, E.M.; Bazighifan, O. An oscillation criterion in 4th-order neutral differential equations with a continuously distributed delay. Adv. Differ. Equ. 2019, 336, 1-9.

10. Chatzarakis, G.E.; Li, T. Oscillation criteria for delay and advanced differential equations with nonmonotone arguments. Complexity 2018, 2018, 8237634. [CrossRef]

11. El-Nabulsi, R.A.; Moaaz, O.; Bazighifan, O. New Results for Oscillatory Behavior of Fourth-Order Differential Equations. Symmetry 2020, 12, 136. [CrossRef]

12. Elabbasy, E.M.; Cesarano, C.; Bazighifan, O.; Moaaz, O. Asymptotic and oscillatory behavior of solutions of a class of higher order differential equation. Symmetry 2019, 11, 1434. [CrossRef]

13. Elabbasy, E.M.; Hassan, T.S.; Moaaz, O. Oscillation behavior of second-order nonlinear neutral differential equations with deviating arguments. Opusc. Math. 2012, 32, 719-730. [CrossRef]

14. Li, T.; Han, Z.; Zhao, P.; Sun, S. Oscillation of even-order neutral delay differential equations. Adv. Differ. Equ. 2010, 127, 503-509.

15. Philos, C.G. On the existence of non-oscillatory solutions tending to zero at $\infty$ for differential equations with positive delays. Arch. Math. 1981, 36, 168-178. [CrossRef]

16. Kiguradze, I.T.; Chanturiya, T.A. Asymptotic Properties of Solutions of Nonautonomous Ordinary Differential Equations; Kluwer Acad. Publ.: Dordrecht, The Netherlands, 1993.

17. Moaaz, O.; Dassios, I.; Bazighifan, O.; Muhib, A. Oscillation Theorems for Nonlinear Differential Equations of Fourth-Order. Mathematics 2020, 8, 520. [CrossRef]

18. Moaaz, O.; Elabbasy, E.M.; Bazighifan, O. On the asymptotic behavior of fourth-order functional differential equations. Adv. Differ. Equ. 2017, 2017, 261. [CrossRef]

19. Moaaz, O.; Elabbasy, E.M.; Muhib, A. Oscillation criteria for even-order neutral differential equations with distributed deviating arguments. Adv. Differ. Equ. 2019, 297, 1-10. [CrossRef]

20. Moaaz, O.; Kumam, P.; Bazighifan, O. On the Oscillatory Behavior of a Class of Fourth-Order Nonlinear Differential Equation. Symmetry 2020, 12, 524. [CrossRef]

21. Minhos, F.; de Sousa, R. Solvability of Coupled Systems of Generalized Hammerstein-Type Integral Equations in the Real Line. Mathematics 2019, 8, 111. [CrossRef]

22. Xing, G.; Li, T.; Zhang, C. Oscillation of higher-order quasi linear neutral differential equations. Adv. Differ. Equ. 2011, 2011, 45. [CrossRef]

23. Zafer, A. Oscillation criteria for even order neutral differential equations. Appl. Math. Lett. 1998, 11, 21-25. [CrossRef]

24. Zhang, Q.; Yan, J. Oscillation behavior of even order neutral differential equations with variable coefficients. Appl. Math. Lett. 2006, 19, 1202-1206. [CrossRef]

25. Grace, S.; Graef, J.; Tunc, E. Oscillatory behavior of a third order neutral dynamice equations with distributed delays. Electron. J. Qual. Theory Differ. Equ. 2016, 14, 1-14.

26. Graef, J.; Grace, S.; Tunc, E. Oscillation criteria for even-order differential equations with unbounded neutral coecients and distributed deviating arguments. Funct. Differ. Equ. 2018, 45, 143-153. 
27. Grace, S.; Graef, J.; Tunc, E. Oscillatory behavior of second order damped neutral differential equations with distributed deviating arguments. Miskolc Math. Notes 2017, 18, 759-769. [CrossRef]

28. Ozdemir, O.; Tunc, E. Asymptotic behavior and oscillation of solutions of third order neutral dynamic equations with distributed deviating arguments. Bull. Math. Anal. Appl. 2018, 10, 31-52.

(C) 2020 by the authors. Licensee MDPI, Basel, Switzerland. This article is an open access article distributed under the terms and conditions of the Creative Commons Attribution (CC BY) license (http://creativecommons.org/licenses/by/4.0/). 